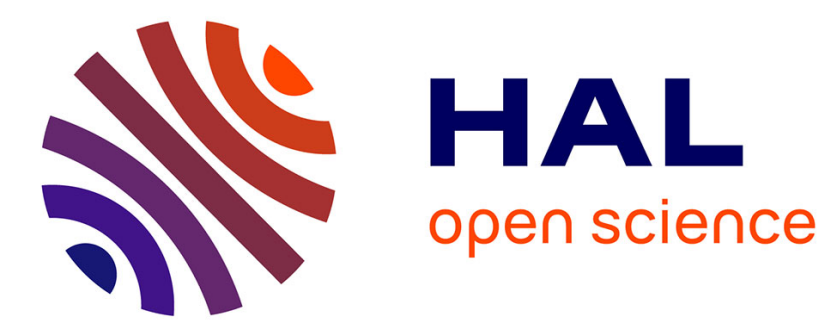

\title{
Nonparametric regression estimation for random fields in a fixed-design
}

\author{
Mohamed El Machkouri
}

\section{To cite this version:}

Mohamed El Machkouri. Nonparametric regression estimation for random fields in a fixed-design. Statistical Inference for Stochastic Processes, 2007. hal-00004096

\section{HAL Id: hal-00004096 https://hal.science/hal-00004096}

Submitted on 4 Feb 2005

HAL is a multi-disciplinary open access archive for the deposit and dissemination of scientific research documents, whether they are published or not. The documents may come from teaching and research institutions in France or abroad, or from public or private research centers.
L'archive ouverte pluridisciplinaire HAL, est destinée au dépôt et à la diffusion de documents scientifiques de niveau recherche, publiés ou non, émanant des établissements d'enseignement et de recherche français ou étrangers, des laboratoires publics ou privés. 


\title{
Nonparametric regression estimation for random fields in a fixed-design
}

\author{
Mohamed EL MACHKOURI
}

4th February 2005

\begin{abstract}
We investigate the nonparametric estimation for regression in a fixed-design setting when the errors are given by a field of dependent random variables. Sufficient conditions for kernel estimators to converge uniformly are obtained. These estimators can attain the optimal rates of uniform convergence and the results apply to a large class of random fields which contains martingale-difference random fields and mixing random fields.
\end{abstract}

AMS Subject Classifications (2000): 60G60, 62G08

Key words and phrases: nonparametric regression estimation, kernel estimators, strong consistency, fixed-design, exponential inequalities, martingale difference random fields, mixing, Orlicz spaces.

Short title: Nonparametric regression in a fixed design. 


\section{Introduction}

Over the last few years nonparametric estimation for random fields (or spatial processes) was given increasing attention stimulated by a growing demand from applied research areas (see Guyon 18]). In fact, spatial data arise in various areas of research including econometrics, image analysis, meterology, geostatistics... Our aim in this paper is to investigate uniform strong convergence rates of a regression estimator in a fixed design setting when the errors are given by a stationary field of dependent random variables which show spatial interaction. We are most interested in conditions which ensure convergence rates to be identical to those in the case of independent errors (see Stone [33]). Currently the author is working on extensions of the present results to the random design framework. Let $\mathbb{Z}^{d}, d \geq 1$ denote the integer lattice points in the $d$-dimensional Euclidean space. By a stationary real random field we mean any family $\left(\varepsilon_{k}\right)_{k \in \mathbb{Z}^{d}}$ of real-valued random variables defined on a probability space $(\Omega, \mathcal{F}, \mathbb{P})$ such that for any $(k, n) \in \mathbb{Z}^{d} \times \mathbb{N}^{*}$ and any $\left(i_{1}, \ldots, i_{n}\right) \in\left(\mathbb{Z}^{d}\right)^{n}$, the random vectors $\left(\varepsilon_{i_{1}}, \ldots, \varepsilon_{i_{n}}\right)$ and $\left(\varepsilon_{i_{1}+k}, \ldots, \varepsilon_{i_{n}+k}\right)$ have the same law. The regression model which we are interested in is

$$
Y_{i}=g(i / n)+\varepsilon_{i}, \quad i \in \Lambda_{n}=\{1, \ldots, n\}^{d}
$$

where $g$ is an unknown smooth function and $\left(\varepsilon_{i}\right)_{i \in \mathbb{Z}^{d}}$ is a zero mean stationary real random field. Note that this model was considered also by Bosq [8] and Hall et Hart [19 for time series $(d=1)$. Let $K$ be a probability kernel defined on $\mathbb{R}^{d}$ and $\left(h_{n}\right)_{n \geq 1}$ a sequence of positive numbers which converges to zero and which satisfies $\left(n h_{n}\right)_{n \geq 1}$ goes to infinity. We estimate the function $g$ by the kernel-type estimator $g_{n}$ defined for any $x$ in $[0,1]^{d}$ by

$$
g_{n}(x)=\frac{\sum_{i \in \Lambda_{n}} Y_{i} K\left(\frac{x-i / n}{h_{n}}\right)}{\sum_{i \in \Lambda_{n}} K\left(\frac{x-i / n}{h_{n}}\right)} .
$$

Note that Assumption A1) in section 2 ensures that $g_{n}$ is well defined. Until now, most of existing theoretical nonparametric results of dependent random variables pertain to time series (see Bosq [9]) and relatively few generalizations to the spatial domain are available. Key references on this topic are Biau [5], Carbon et al. [10], Carbon et al. [11], Hallin et al. [20], [21], Tran [34, Tran and Yakowitz [35] and Yao [36] who have investigated nonparametric density estimation for random fields and Altman [2], Biau and Cadre [6], Hallin et al. [22] and $\mathrm{Lu}$ and Chen [25], [26] who have studied spatial prediction and spatial regression estimation. The classical asymptotic theory in statistics is built upon central limit theorems, law of large numbers and 
large deviations inequalities for the sequences of random variables. These classical limit theorems have been extended to the setting of spatial processes. In particular, some key results on the central limit theorem and its functional versions are Alexander and Pyke [1], Bass [3], Basu and Dorea [4, Bolthausen [7] and more recently Dedecker [12], [13], El Machkouri [16] and El Machkouri and Volný [17]. For a survey on limit theorems for spatial processes and some applications in statistical physics, one can refer to Nahapetian [28]. Note also that the main results (section 3) of this work are obtained via exponential inequalities for random fields discovered by $\mathrm{El}$ Machkouri [16].

The paper is organized as follows. The next section sets up the notations and the assumptions which will be considered in the sequel. In section 3, we present our main results on both weak and strong consistencies rates of the estimator $g_{n}$. The last section is devoted to the proofs.

\section{Notations and Assumptions}

In the sequel we denote $\|x\|=\max _{1 \leq k \leq d}\left|x_{k}\right|$ for any $x=\left(x_{1}, \ldots, x_{d}\right) \in[0,1]^{d}$. With a view to obtain optimal convergence rates for the estimator $g_{n}$ defined by (2), we have to make the following assumptions on the regression function $g$ and the probability kernel $\mathrm{K}$ :

A1) The probability kernel $K$ is symmetric, nonnegative, supported by $[-1,1]^{d}$ and satisfies a Lipschitz condition $|K(x)-K(y)| \leq \eta\|x-y\|$ for any $x, y \in[-1,1]^{d}$ and some $\eta>0$. In addition there exists $c, C>0$ such that $c \leq K(x) \leq C$ for any $x \in[-1,1]^{d}$.

A2) There exists a constant $B>0$ such that $|g(x)-g(y)| \leq B\|x-y\|$ for any $x, y \in[0,1]^{d}$, that is $g$ is $B$-Lipschitz.

A Young function $\psi$ is a real convex nondecreasing function defined on $\mathbb{R}^{+}$ which satisfies $\lim _{t \rightarrow \infty} \psi(t)=+\infty$ and $\psi(0)=0$. We define the Orlicz space $L_{\psi}$ as the space of real random variables $Z$ defined on the probability space $(\Omega, \mathcal{F}, \mathbb{P})$ such that $E[\psi(|Z| / c)]<+\infty$ for some $c>0$. The Orlicz space $L_{\psi}$ equipped with the so-called Luxemburg norm $\|\cdot\|_{\psi}$ defined for any real random variable $Z$ by

$$
\|Z\|_{\psi}=\inf \{c>0 ; E[\psi(|Z| / c)] \leq 1\}
$$

is a Banach space. For more about Young functions and Orlicz spaces one can refer to Krasnosel'skii and Rutickii [24]. Let $\beta>0$. We denote by $\psi_{\beta}$ 
the Young function defined for any $x \in \mathbb{R}^{+}$by

$$
\psi_{\beta}(x)=\exp \left(\left(x+\xi_{\beta}\right)^{\beta}\right)-\exp \left(\xi_{\beta}^{\beta}\right) \quad \text { where } \quad \xi_{\beta}=((1-\beta) / \beta)^{1 / \beta} \mathbb{1}_{\{0<\beta<1\}} .
$$

On the lattice $\mathbb{Z}^{d}$ we define the lexicographic order as follows: if $i=\left(i_{1}, \ldots, i_{d}\right)$ and $j=\left(j_{1}, \ldots, j_{d}\right)$ are distinct elements of $\mathbb{Z}^{d}$, the notation $i<_{\text {lex }} j$ means that either $i_{1}<j_{1}$ or for some $p$ in $\{2,3, \ldots, d\}, i_{p}<j_{p}$ and $i_{q}=j_{q}$ for $1 \leq q<p$. Let the sets $\left\{V_{i}^{k} ; i \in \mathbb{Z}^{d}, k \in \mathbb{N}^{*}\right\}$ be defined as follows:

$$
V_{i}^{1}=\left\{j \in \mathbb{Z}^{d} ; j<_{\text {lex }} i\right\}
$$

and for $k \geq 2$

$$
V_{i}^{k}=V_{i}^{1} \cap\left\{j \in \mathbb{Z}^{d} ;|i-j| \geq k\right\} \quad \text { where } \quad|i-j|=\max _{1 \leq l \leq d}\left|i_{l}-j_{l}\right| .
$$

For any subset $\Gamma$ of $\mathbb{Z}^{d}$ define $\mathcal{F}_{\Gamma}=\sigma\left(\varepsilon_{i} ; i \in \Gamma\right)$ and set

$$
E_{|k|}\left(\varepsilon_{i}\right)=E\left(\varepsilon_{i} \mid \mathcal{F}_{V_{i}^{|k|}}\right), \quad k \in V_{i}^{1} .
$$

Denote $\beta(q)=2 q /(2-q)$ for $0<q<2$ and consider the following conditions:

C1) $\varepsilon_{0} \in L^{\infty}$ and

$$
\sum_{k \in V_{0}^{1}}\left\|\varepsilon_{k} E_{|k|}\left(\varepsilon_{0}\right)\right\|_{\infty}<\infty
$$

C2) There exists $0<q<2$ such that $\varepsilon_{0} \in L_{\psi_{\beta(q)}}$ and

$$
\sum_{k \in V_{0}^{1}}\left\|\sqrt{\left|\varepsilon_{k} E_{|k|}\left(\varepsilon_{0}\right)\right|}\right\|_{\psi_{\beta(q)}}^{2}<\infty .
$$

C3) There exists $p>2$ such that $\varepsilon_{0} \in L^{p}$ and

$$
\sum_{k \in V_{0}^{1}}\left\|\varepsilon_{k} E_{|k|}\left(\varepsilon_{0}\right)\right\|_{\frac{p}{2}}<\infty
$$

C4) $\varepsilon_{0} \in L^{2}$ and $\sum_{k \in \mathbb{Z}^{d}}\left|E\left(\varepsilon_{0} \varepsilon_{k}\right)\right|<\infty$.

Remark 1 Note that Dedecker [12] established the central limit theorem for any stationary square-integrable random field $\left(\varepsilon_{k}\right)_{k \in \mathbb{Z}^{d}}$ which satisfies the condition $\sum_{k \in V_{0}^{1}}\left\|\varepsilon_{k} E_{|k|}\left(\varepsilon_{0}\right)\right\|_{1}<\infty$. 
In classical statistical physics, there exists spatial processes which satisfy conditions C1),..,C4). For example, Nahapetian and Petrosian [29] gave sufficient conditions for a Gibbs field $\left(\varepsilon_{k}\right)_{k \in \mathbb{Z}^{d}}$ to possess the following martingale difference property: for any $i$ in $\mathbb{Z}^{d}, E\left(\varepsilon_{i} \mid \mathcal{F}_{V_{i}^{1}}\right)=0$ a.s. Another examples of random fields which satisfy conditions $\mathbf{C 1}), \ldots, \mathbf{C 4})$ can be found also among the class of mixing random fields. More precisely, given two sub- $\sigma$-algebras $\mathcal{U}$ and $\mathcal{V}$ of $\mathcal{F}$, different measures of their dependence have been considered in the literature. We are interested by two of them. The $\alpha$-mixing and $\phi$-mixing coefficients had been introduced by Rosenblatt 31] and Ibragimov 23] respectively and can be defined by

$$
\begin{aligned}
& \alpha(\mathcal{U}, \mathcal{V})=\sup \{|\mathbb{P}(U \cap V)-\mathbb{P}(U) \mathbb{P}(V)|, U \in \mathcal{U}, V \in \mathcal{V}\} \\
& \phi(\mathcal{U}, \mathcal{V})=\sup \left\{\|\mathbb{P}(V \mid \mathcal{U})-\mathbb{P}(V)\|_{\infty}, V \in \mathcal{V}\right\} .
\end{aligned}
$$

We have $2 \alpha(\mathcal{U}, \mathcal{V}) \leq \phi(\mathcal{U}, \mathcal{V})$ and these coefficients equal zero if and only if the $\sigma$-algebras $\mathcal{U}$ and $\mathcal{V}$ are independent. Denote by $\sharp \Gamma$ the cardinality of any subset $\Gamma$ of $\mathbb{Z}^{d}$. In the sequel, we shall use the following non-uniform mixing coefficients defined for any $(k, l, n)$ in $\left(\mathbb{N}^{*} \cup\{\infty\}\right)^{2} \times \mathbb{N}$ by

$$
\begin{aligned}
& \alpha_{k, l}(n)=\sup \left\{\alpha\left(\mathcal{F}_{\Gamma_{1}}, \mathcal{F}_{\Gamma_{2}}\right), \sharp \Gamma_{1} \leq k, \sharp \Gamma_{2} \leq l, \rho\left(\Gamma_{1}, \Gamma_{2}\right) \geq n\right\}, \\
& \phi_{k, l}(n)=\sup \left\{\phi\left(\mathcal{F}_{\Gamma_{1}}, \mathcal{F}_{\Gamma_{2}}\right), \sharp \Gamma_{1} \leq k, \sharp \Gamma_{2} \leq l, \rho\left(\Gamma_{1}, \Gamma_{2}\right) \geq n\right\},
\end{aligned}
$$

where the distance $\rho$ is defined by $\rho\left(\Gamma_{1}, \Gamma_{2}\right)=\min \left\{|i-j|, i \in \Gamma_{1}, j \in \Gamma_{2}\right\}$. We say that the random field $\left(\varepsilon_{k}\right)_{k \in \mathbb{Z}^{d}}$ is $\alpha$-mixing or $\phi$-mixing if there exists a pair $(k, l)$ in $\left(\mathbb{N}^{*} \cup\{\infty\}\right)^{2}$ such that $\lim _{n \rightarrow \infty} \alpha_{k, l}(n)=0$ or $\lim _{n \rightarrow \infty} \phi_{k, l}(n)=0$ respectively. For more about mixing coefficients one can refer to Doukhan [15]. We consider the following mixing conditions:

$\mathbf{C}^{\prime}$ 1) $\varepsilon_{0} \in L^{\infty}$ and

$$
\sum_{k \in \mathbb{Z}^{d}} \phi_{\infty, 1}(|k|)<\infty
$$

$\left.\mathbf{C}^{\prime} 2\right)$ There exists $0<q<2$ such that $\varepsilon_{0} \in L_{\psi_{\beta(q)}}$ and

$$
\sum_{k \in \mathbb{Z}^{d}} \sqrt{\phi_{\infty, 1}(|k|)}<\infty
$$

or

$$
\sum_{k \in \mathbb{Z}^{d}} c_{k}^{2}(\beta(q))<\infty
$$

where for any $\beta>0$

$$
c_{k}(\beta)=\inf \left\{c>0 \mid \int_{0}^{\alpha_{1, \infty}(|k|)} \psi_{\beta}\left(\frac{Q_{\varepsilon_{0}}(u)}{c}\right) d u \leq 1\right\} .
$$


$\mathbf{C}^{\prime}$ 3) There exists $p>2$ such that $\varepsilon_{0} \in L^{p}$ and

$$
\sum_{k \in \mathbb{Z}^{d}}\left(\int_{0}^{\alpha_{1, \infty}(|k|)} Q_{\varepsilon_{0}}^{p}(u) d u\right)^{2 / p}<\infty
$$

where $Q_{\varepsilon_{0}}$ is the inverse cadlag of the tail function $t \rightarrow \mathbb{P}\left(\left|\varepsilon_{0}\right|>t\right)$ (i.e. for any $\left.u \geq 0, Q_{\varepsilon_{0}}(u)=\inf \left\{t>0 \mid \mathbb{P}\left(\left|\varepsilon_{0}\right|>t\right) \leq u\right\}\right)$.

Remark 2 Let us note that if $p=2+\delta$ for some $\delta>0$ then the condition

$$
\sum_{m=1}^{\infty} m^{d-1} \alpha_{1, \infty}^{\frac{\delta}{2+\delta}-\varepsilon}(m)<\infty \quad \text { for some } \varepsilon>0
$$

is more restrictive than condition (国) and is known to be sufficient for the random field $\left(\varepsilon_{k}\right)_{k \in \mathbb{Z}^{d}}$ to satisfy a functional central limit theorem (cf. Dedecker [13]).

In statistical physics, using the Dobrushin's uniqueness condition (cf. [14]), one can construct Gibbs fields satisfying a uniform exponential mixing condition which is more restrictive than conditions $\mathbf{C}^{\prime} \mathbf{1}$ ), $\mathbf{C}^{\prime} \mathbf{2}$ ) and $\mathbf{C}^{\prime} \mathbf{3}$ ) (see Guyon [18, theorem 2.1.3, p. 52).

\section{Main results}

Let $\left(Z_{n}\right)_{n \geq 1}$ be a sequence of real random variables and $\left(v_{n}\right)_{n \geq 1}$ be a sequence of positive numbers. We say that

$$
Z_{n}=O_{a . s .}\left[v_{n}\right]
$$

if there exists $\lambda>0$ such that

$$
\limsup _{n \rightarrow \infty} \frac{\left|Z_{n}\right|}{v_{n}} \leq \lambda \quad \text { a.s. }
$$

Our main result is the following.

Theorem 1 Assume that the assumption A1) holds.

1) If $\mathbf{C 1 ) ~ h o l d s ~ t h e n ~}$

$$
\sup _{x \in[0,1]^{d}}\left|g_{n}(x)-E g_{n}(x)\right|=O_{a . s .}\left[\frac{(\log n)^{1 / 2}}{\left(n h_{n}\right)^{d / 2}}\right] \text {. }
$$


2) If C2) holds for some $0<q<2$ then

$$
\sup _{x \in[0,1]^{d}}\left|g_{n}(x)-E g_{n}(x)\right|=O_{a . s .}\left[\frac{(\log n)^{1 / q}}{\left(n h_{n}\right)^{d / 2}}\right] \text {. }
$$

3) Assume that C3) holds for some $p>2$ and $h_{n}=n^{-\theta_{2}}(\log n)^{\theta_{1}}$ for some $\theta_{1}, \theta_{2}>0$. Let $a, b \geq 0$ be fixed and denote

$$
v_{n}=\frac{n^{a}(\log n)^{b}}{\left(n h_{n}\right)^{d / 2}} \quad \text { and } \quad \theta=\frac{2 a(d+p)-d^{2}-2}{d(3 d+2)} .
$$

If $\theta \geq \theta_{2}$ and $d(3 d+2) \theta_{1}+2(d+p) b>2$ then

$$
\sup _{x \in[0,1]^{d}}\left|g_{n}(x)-E g_{n}(x)\right|=O_{a . s .}\left[v_{n}\right] .
$$

Remark 3 Theorem 1 shows that the optimal uniform convergence rate is obtained for bounded errors (cf. estimation (5)) and that it is "almost" optimal if one considers errors with only finite exponential moments (cf. estimation (6)).

Theorem 2 Assume that the assumption A1) holds.

1) Assume that C3) holds for some $p>2$. Let $a>0$ be fixed and denote

$$
v_{n}=\frac{n^{a}}{\left(n h_{n}\right)^{d / 2}} \quad \text { and } \quad \theta=\frac{2 a(d+p)-d^{2}}{d(3 d+2)} .
$$

If $\theta>0$ and $h_{n} \geq n^{-\theta}$ then

$$
\left\|\sup _{x \in[0,1]^{d}}\left|g_{n}(x)-E g_{n}(x)\right|\right\|_{p}=O\left[v_{n}\right] .
$$

2) If C4) holds then

$$
\sup _{x \in[0,1]^{d}}\left\|g_{n}(x)-E g_{n}(x)\right\|_{2}=O\left[\left(n h_{n}\right)^{-d / 2}\right] .
$$

In the sequel, we denote by $\operatorname{Lip}(B)$ the set of $B$-Lipschitz functions. The following proposition gives the convergence of $E g_{n}(x)$ to $g(x)$.

Proposition 1 Assume that the assumption A2) holds then

$$
\sup _{x \in[0,1]^{d}} \sup _{g \in \operatorname{Lip}(B)}\left|E g_{n}(x)-g(x)\right|=O\left[h_{n}\right] .
$$


From Proposition [ ] and Theorem [ ] we derive the following corollary.

Corollary 1 Assume that A1) and A2) hold and let $h_{n}=\left(n^{-d} \log n\right)^{1 /(2+d)}$.

1) If $\mathbf{C 1 ) ~ h o l d s ~ t h e n ~}$

$$
\sup _{x \in[0,1]^{d}} \sup _{g \in L i p(B)}\left|g_{n}(x)-g(x)\right|=O_{a . s .}\left[\left(\frac{\log n}{n^{d}}\right)^{\frac{1}{2+d}}\right] .
$$

2) If C2) holds for some $0<q<2$ then

$$
\sup _{x \in[0,1]^{d}} \sup _{g \in \operatorname{Lip}(B)}\left|g_{n}(x)-g(x)\right|=O_{a . s .}\left[u(n)\left(\frac{\log n}{n^{d}}\right)^{\frac{1}{2+d}}\right]
$$

where $u(n)=(\log n)^{(2-q) / 2 q}$.

3) Let $\varepsilon>0$ be fixed. If C3) holds for some $p>2$ satisfying

$$
p \geq \frac{4 d^{3}+(4-2 \varepsilon) d^{2}+(2-4 \varepsilon) d+4}{2 \varepsilon(2+d)}
$$

then

$$
\sup _{x \in[0,1]^{d}} \sup _{g \in \operatorname{Lip}(B)}\left|g_{n}(x)-g(x)\right|=O_{\text {a.s. }}\left[u(n)\left(\frac{\log n}{n^{d}}\right)^{\frac{1}{2+d}}\right]
$$

where $u(n)=n^{\varepsilon}$.

Remark 4 Note that the consistency rate $\left(n^{-d} \log n\right)^{1 /(2+d)}$ is known to be the optimal one (see Stone [33]).

From Proposition 1 and Theorem 2 we derive the following corollary.

Corollary 2 Assume that A1) and A2) hold and let $h_{n}=n^{-d /(2+d)}$.

1) Let $\varepsilon>0$ be fixed. If C3) holds for some $p>2$ satisfying

$$
p \geq \frac{4 d^{3}+(4-2 \varepsilon) d^{2}-4 \varepsilon d}{2 \varepsilon(2+d)}
$$

then

$$
\left\|\sup _{x \in[0,1]^{d}} \sup _{g \in \operatorname{Lip}(B)}\left|g_{n}(x)-g(x)\right|\right\|_{p}=O\left[n^{-\frac{d}{2+d}+\varepsilon}\right] .
$$


2) If $\mathbf{C 4 ) ~ h o l d s ~ t h e n ~}$

$$
\sup _{x \in[0,1]^{d}}\left\|\sup _{g \in \operatorname{Lip}(B)}\left|g_{n}(x)-g(x)\right|\right\|_{2}=O\left[n^{-\frac{d}{2+d}}\right] .
$$

Finally the rates of convergence obtained above are valid when the errors are given by a mixing random field. More precisely, we have the following corollary.

Corollary 3 Theorems 1 and 2 and Corollaries 11 and 2 still hold if one replace conditions $\mathbf{C 1}$ ), C2) and $\mathbf{C 3}$ ) by conditions $\left.\mathbf{C}^{\prime} \mathbf{1}\right), \mathbf{C}^{\prime} \mathbf{2}$ ) and $\mathbf{C}^{\prime} \mathbf{3}$ ) respectively.

\section{Proofs}

For any $x$ in $[0,1]^{d}$ and any integer $n \geq 1$ we define $B_{n}(x)=E g_{n}(x)-g(x)$ and $V_{n}(x)=g_{n}(x)-E g_{n}(x)$. More precisely

$$
\begin{aligned}
B_{n}(x) & =\frac{\sum_{i \in \Lambda_{n}} a_{i}(x) g(i / n)}{\sum_{i \in \Lambda_{n}} a_{i}(x)}-g(x) \\
V_{n}(x) & =\frac{\sum_{i \in \Lambda_{n}} a_{i}(x) \varepsilon_{i}}{\sum_{i \in \Lambda_{n}} a_{i}(x)}
\end{aligned}
$$

where $a_{i}(x)=K\left(\frac{x-i / n}{h_{n}}\right)$. In the sequel, we denote also $S_{n}(x)=\sum_{i \in \Lambda_{n}} a_{i}(x) \varepsilon_{i}$ for any $x \in[0,1]^{d}$. We start with the following lemma.

Lemma 1 There exists constants $c, C>0$ such that for any $x \in[0,1]^{d}$ and any $n \in \mathbb{N}^{*}$,

$$
c \prod_{k=1}^{d}\left[n\left(x_{k}+h_{n}\right)\right] \leq \sum_{i \in \Lambda_{n}} a_{i}(x) \leq C \prod_{k=1}^{d}\left[n\left(x_{k}+h_{n}\right)\right]
$$

where [.] denote the integer part function.

Proof of Lemma 1. Since the kernel $K$ is supported by $[-1,1]^{d}$, we have

$$
\sum_{i \in \Lambda_{n}} a_{i}(x)=\sum_{i_{1}=1}^{\left[n\left(x_{1}+h_{n}\right)\right]} \cdots \sum_{i_{d}=1}^{\left[n\left(x_{d}+h_{n}\right)\right]} a_{i}(x) .
$$

By assumption, there exists constants $c, C>0$ such that $c \leq K(y) \leq C$ for any $y \in[-1,1]^{d}$. The proof of Lemma 1 is complete. 


\subsection{Proof of Theorem 1}

Let $\left(v_{n}\right)_{n \geq 1}$ be a sequence of positive numbers going to zero. Following Carbon and al. [11] the compact set $[0,1]^{d}$ can be covered by $r_{n}$ cubes $I_{k}$ having sides of length $l_{n}=v_{n} h_{n}^{2 d+1}$ and center at $c_{k}$. Clearly there exists $c>0$ such that $r_{n} \leq c / l_{n}^{d}$. Define

$$
\begin{aligned}
A_{1, n}(g) & =\max _{1 \leq k \leq r_{n}} \sup _{x \in I_{k}}\left|g_{n}(x)-g_{n}\left(c_{k}\right)\right| \\
A_{2, n}(g) & =\max _{1 \leq k \leq r_{n}} \sup _{x \in I_{k}}\left|E g_{n}(x)-E g_{n}\left(c_{k}\right)\right| \\
A_{3, n} & =\max _{1 \leq k \leq r_{n}}\left|g_{n}\left(c_{k}\right)-E g_{n}\left(c_{k}\right)\right|
\end{aligned}
$$

then

$$
\sup _{x \in[0,1]^{d}}\left|g_{n}(x)-E g_{n}(x)\right| \leq \sup _{g \in \operatorname{Lip}(B)}\left[A_{1, n}(g)+A_{2, n}(g)\right]+A_{3, n} .
$$

Lemma 2 For $i=1,2$ we have

$$
\sup _{g \in \operatorname{Lip}(B)} A_{i, n}(g)=O_{a . s .}\left[v_{n}\right]
$$

Proof of Lemma 2. Since $g \in \operatorname{Lip}(B)$, we can assume without loss of generality that $g$ is bounded by $B$ on the set $[0,1]^{d}$. For any $x \in I_{k}$, we have

$$
g_{n}(x)-g_{n}\left(c_{k}\right)=\sigma_{1}+\sigma_{2}
$$

where

$$
\sigma_{1}=\frac{\sum_{i \in \Lambda_{n}} Y_{i}\left(a_{i}(x)-a_{i}\left(c_{k}\right)\right)}{\sum_{i \in \Lambda_{n}} a_{i}(x)}
$$

and

$$
\sigma_{2}=\frac{\sum_{i \in \Lambda_{n}}\left(a_{i}\left(c_{k}\right)-a_{i}(x)\right)}{\sum_{i \in \Lambda_{n}} a_{i}(x) \times \sum_{i \in \Lambda_{n}} a_{i}\left(c_{k}\right)} \sum_{i \in \Lambda_{n}} Y_{i} a_{i}\left(c_{k}\right) .
$$

Now, by Lemma 1 and Assumption A1), we derive that there exists constants $c, \eta>0$ such that for any $n$ sufficiently large

$$
\left|\sigma_{1}\right| \leq \frac{2^{d} \eta l_{n} / h_{n}}{c\left(n h_{n}\right)^{d}} \sum_{i \in \Lambda_{n}}\left|Y_{i}\right| \leq \frac{\eta v_{n} h_{n}^{d}}{c}\left(B+\frac{1}{n^{d}} \sum_{i \in \Lambda_{n}}\left|\varepsilon_{i}\right|\right)
$$

and

$$
\left|\sigma_{2}\right| \leq \frac{4^{d} \eta n^{d} l_{n} / h_{n}}{c^{2}\left(n h_{n}\right)^{2 d}} \sum_{i \in \Lambda_{n}}\left|Y_{i}\right| \leq \frac{\eta v_{n}}{c^{2}}\left(B+\frac{1}{n^{d}} \sum_{i \in \Lambda_{n}}\left|\varepsilon_{i}\right|\right)
$$


Since $\left(\varepsilon_{i}\right)$ is a stationary ergodic random field the lemma easily follows from the last inequalities and the Birkhoff ergodic theorem. The proof of Lemma 2 is complete.

Lemma 3 Assume that either C1) holds and $v_{n}=(\log n)^{1 / 2} /\left(n h_{n}\right)^{d / 2}$ or C2) holds for some $0<q<2$ and $v_{n}=(\log n)^{1 / q} /\left(n h_{n}\right)^{d / 2}$ then

$$
A_{3, n}=O_{\text {a.s. }}\left[v_{n}\right]
$$

Proof of Lemma 3. Let $0<q \leq 2$ be fixed. We consider the exponential Young function define for any $x \in \mathbb{R}^{+}$by $\psi_{q}(x)=\exp \left(\left(x+\xi_{q}\right)^{q}\right)-\exp \left(\xi_{q}^{q}\right)$ where $\xi_{q}=((1-q) / q)^{1 / q} \mathbb{1}_{\{0<q<1\}}$. Let $\lambda>0$ and $x \in[0,1]^{d}$ be fixed

$$
\begin{aligned}
\mathbb{P}\left(\left|V_{n}(x)\right|>\lambda v_{n}\right) & =\mathbb{P}\left(\left|S_{n}(x)\right|>\lambda v_{n} \sum_{i \in \Lambda_{n}} a_{i}(x)\right) \\
& \leq\left(1+e^{\xi_{q}^{q}}\right) \exp \left[-\left(\frac{\lambda v_{n} \sum_{i \in \Lambda_{n}} a_{i}(x)}{\left\|\sum_{i \in \Lambda_{n}} a_{i}(x) \varepsilon_{i}\right\|_{\psi_{q}}}+\xi_{q}\right)^{q}\right] .
\end{aligned}
$$

For any $i \in \Lambda_{n}$ and any $0<q<2$ denote

$$
b_{i, q}(a(x) \varepsilon)=\left\|a_{i}(x) \varepsilon_{i}\right\|_{\psi_{\beta(q)}}^{2}+\sum_{k \in V_{i}^{1}}\left\|\sqrt{\left|a_{k}(x) \varepsilon_{k} E_{|k-i|}\left(a_{i}(x) \varepsilon_{i}\right)\right|}\right\|_{\psi_{\beta(q)}}^{2}
$$

and

$$
b_{i, 2}(a(x) \varepsilon)=\left\|a_{i}(x) \varepsilon_{i}\right\|_{\infty}^{2}+\sum_{k \in V_{i}^{1}}\left\|a_{k}(x) \varepsilon_{k} E_{|k-i|}\left(a_{i}(x) \varepsilon_{i}\right)\right\|_{\infty}
$$

where $V_{i}^{1}=\left\{j \in \mathbb{Z}^{d} ; j<_{\text {lex }} i\right\}$. Using Kahane-Khintchine inequalities (cf. El Machkouri [16], Theorem 1) we derive that if Condition C2) holds for some $0<q<2$ then

$$
\mathbb{P}\left(\left|V_{n}(x)\right|>\lambda v_{n}\right) \leq\left(1+e^{\xi_{q}^{q}}\right) \exp \left[-\left(\frac{\lambda v_{n} \sum_{i \in \Lambda_{n}} a_{i}(x)}{M\left(\sum_{i \in \Lambda_{n}} b_{i, q}(a(x) \varepsilon)\right)^{1 / 2}}+\xi_{q}\right)^{q}\right]
$$

where $M$ is a positive constant depending only on $q$ and on the probability kernel $K$. Now using the definition (19) and Lemma 11 there exist constants $c, M>0$ such that

$$
\begin{aligned}
\sup _{x \in[0,1]^{d}} \mathbb{P}\left(\left|V_{n}(x)\right|>\lambda v_{n}\right) & \leq\left(1+e^{\xi_{q}^{q}}\right) \exp \left[-\left(\frac{\lambda v_{n}\left(\sum_{i \in \Lambda_{n}} a_{i}(x)\right)^{1 / 2}}{M}+\xi_{q}\right)^{q}\right] \\
& \leq\left(1+e^{\xi_{q}^{q}}\right) \exp \left[-\frac{c^{q} \lambda^{q} v_{n}^{q}\left(\left[n h_{n}\right]\right)^{d q / 2}}{M^{q}}\right]
\end{aligned}
$$


So if $v_{n}=(\log n)^{1 / q} /\left(n h_{n}\right)^{d / 2}$ and $n$ is sufficiently large then

$$
\sup _{x \in[0,1]^{d}} \mathbb{P}\left(\left|V_{n}(x)\right|>\lambda v_{n}\right) \leq\left(1+e^{\xi_{q}^{q}}\right) \exp \left[-\frac{c^{q} \lambda^{q} \log n}{2^{d q / 2} M^{q}}\right] .
$$

If Condition C1) holds then (21) still hold with $q=2$ (cf. El Machkouri 16, Theorem 1). So if $v_{n}=(\log n)^{1 / 2} /\left(n h_{n}\right)^{d / 2}$ and $n$ is large it follows that

$$
\sup _{x \in[0,1]^{d}} \mathbb{P}\left(\left|V_{n}(x)\right|>\lambda v_{n}\right) \leq 2 \exp \left[-\frac{c^{2} \lambda^{2} \log n}{2^{d} M^{2}}\right] .
$$

Since

$$
\mathbb{P}\left(\left|A_{3, n}\right|>\lambda v_{n}\right) \leq r_{n} \sup _{x \in[0,1]^{d}} \mathbb{P}\left(\left|V_{n}(x)\right|>\lambda v_{n}\right),
$$

using (22) and (23), choosing $\lambda$ sufficiently large and applying Borel-Cantelli's lemma, we derive

$$
\mathbb{P}\left(\limsup _{n \rightarrow \infty}\left\{\left|A_{3, n}\right|>\lambda v_{n}\right\}\right)=0
$$

and

$$
\mathbb{P}\left(\limsup _{n \rightarrow \infty} \frac{\left|A_{3, n}\right|}{v_{n}} \leq \lambda\right)=1 .
$$

The proof of points 1) and 2) of Theorem 1 are completed by combining Inequality (18) with Lemmas 2 and 3 .

Lemma 4 Assume that C3) holds for some $p>2$ and $h_{n}=n^{-\theta_{2}}(\log n)^{\theta_{1}}$ for some $\theta_{1}, \theta_{2}>0$. Let $a, b \geq 0$ be fixed and denote

$$
v_{n}=\frac{n^{a}(\log n)^{b}}{\left(n h_{n}\right)^{d / 2}} \quad \text { and } \quad \theta=\frac{2 a(d+p)-d^{2}-2}{d(3 d+2)} .
$$

If $\theta \geq \theta_{2}$ and $d(3 d+2) \theta_{1}+2(d+p) b>2$ then

$$
\lim _{n \rightarrow+\infty} \frac{\left|A_{3, n}\right|}{v_{n}}=0 \quad \text { a.s. }
$$

Proof of Lemma 4 . Let $p>2$ be fixed. For any $\lambda>0$

$$
\begin{aligned}
\mathbb{P}\left(\left|V_{n}(x)\right|>\lambda v_{n}\right) & =\mathbb{P}\left(\left|S_{n}(x)\right|>\lambda v_{n} \sum_{i \in \Lambda_{n}} a_{i}(x)\right) \\
& \leq \frac{\lambda^{-p} E\left|S_{n}(x)\right|^{p}}{v_{n}^{p}\left(\sum_{i \in \Lambda_{n}} a_{i}(x)\right)^{p}} \\
& \leq \frac{\lambda^{-p}}{v_{n}^{p}\left(\sum_{i \in \Lambda_{n}} a_{i}(x)\right)^{p}}\left(2 p \sum_{i \in \Lambda_{n}} c_{i}(x)\right)^{p / 2}
\end{aligned}
$$


where $c_{i}(x)=a_{i}(x)^{2}\left\|\varepsilon_{i}\right\|_{p}^{2}+a_{i}(x) \sum_{k \in V_{i}^{1}} a_{k}(x)\left\|\varepsilon_{k} E_{|k-i|}\left(\varepsilon_{i}\right)\right\|_{\frac{p}{2}}$. The last estimate follows from a Marcinkiewicz-Zygmund type inequality by Dedecker (see [13]) for real random fields. Noting that there exists $\gamma>0$ such that $c_{i}(x) \leq \gamma a_{i}(x), x \in[0,1]^{d}$ and using Lemma 1, we derive that there exists $\gamma^{\prime}>0$ such that

$$
\mathbb{P}\left(\left|A_{3, n}\right|>\lambda v_{n}\right) \leq r_{n} \sup _{x \in[0,1]^{d}} \mathbb{P}\left(\left|V_{n}(x)\right|>\lambda v_{n}\right) \leq \frac{\gamma^{\prime}}{\tau_{n} \lambda^{p}}
$$

where $\tau_{n}=l_{n}^{d} v_{n}^{p}\left(\left[n h_{n}\right]\right)^{d p / 2}$. Since $v_{n}=n^{a}(\log n)^{b} /\left(n h_{n}\right)^{d / 2}$ and $l_{n}=v_{n} h_{n}^{2 d+1}$ it follows

$$
\frac{1}{\tau_{n}}=\frac{\left(n h_{n}\right)^{d(d+p) / 2}}{h_{n}^{d(2 d+1)} n^{a(d+p)}(\log n)^{b(d+p)}\left(\left[n h_{n}\right]\right)^{d p / 2}} .
$$

If $n$ is sufficiently large, we derive

$$
\begin{aligned}
\frac{1}{\tau_{n}} & \leq \frac{2^{d p / 2}\left(n h_{n}\right)^{d(d+p) / 2}}{h_{n}^{d(2 d+1)} n^{a(d+p)}(\log n)^{b(d+p)}\left(n h_{n}\right)^{d p / 2}} \\
& =\frac{2^{d p / 2}}{h_{n}^{d(3 d+2) / 2} n^{a(d+p)-d^{2} / 2}(\log n)^{b(d+p)}} \\
& \leq \frac{2^{d p / 2}}{n(\log n)^{b(d+p)+\theta_{1} d(3 d+2) / 2}} \quad \text { since } \theta \geq \theta_{2} .
\end{aligned}
$$

Now $b(d+p)+\theta_{1} d(3 d+2) / 2>1$ implies $\sum_{n \geq 1} \tau_{n}^{-1}<\infty$. Applying BorelCantelli's lemma, it follows that for any $\lambda>0$

$$
\mathbb{P}\left(\limsup _{n \rightarrow \infty}\left\{\left|A_{3, n}\right|>\lambda v_{n}\right\}\right)=0
$$

that is for any $\lambda>0$

$$
\mathbb{P}\left(\limsup _{n \rightarrow \infty} \frac{\left|A_{3, n}\right|}{v_{n}} \leq \lambda\right)=1
$$

The proof of Lemma 1 is complete and the point 3 ) of Theorem 1 is obtained by combining Inequality (18) with Lemmas 2 and 4 . The proof of Theorem 1 is complete.

\subsection{Proof of Theorem 2}

We follow the first part of the proof of Theorem 1 and we consider the estimation (18). 
Lemma 5 Assume that C3) holds for some $p>2$. Let $a>0$ be fixed and denote

$$
v_{n}=\frac{n^{a}}{\left(n h_{n}\right)^{d / 2}} \quad \text { and } \quad \theta=\frac{2 a(d+p)-d^{2}}{d(3 d+2)} .
$$

If $\theta>0$ and $h_{n} \geq n^{-\theta}$ then

$$
\left\|A_{3, n}\right\|_{p}=O\left[v_{n}\right] .
$$

Proof of Lemma 5. Let $p>2$ and $x \in[0,1]^{d}$ be fixed. Using the MarcinkiewiczZygmund type inequality by Dedecker (see [13]) as in the proof of Lemma 1 there exist $\gamma^{\prime \prime}, c>0$ such that

$$
\begin{aligned}
\left\|V_{n}(x)\right\|_{p} & =\left(\frac{E\left|S_{n}(x)\right|^{p}}{\left(\sum_{i \in \Lambda_{n}} a_{i}(x)\right)^{p}}\right)^{1 / p} \\
& \leq \gamma^{\prime \prime}\left(\sum_{i \in \Lambda_{n}} a_{i}(x)\right)^{-1 / 2} \\
& \leq \frac{\gamma^{\prime \prime}}{\sqrt{c}}\left(\left[n h_{n}\right]\right)^{-d / 2} \quad \text { by Lemma [1 }
\end{aligned}
$$

It follows that

$$
r_{n}^{1 / p} \sup _{x \in[0,1]^{d}}\left\|V_{n}(x)\right\|_{p}=O\left[\frac{v_{n}}{\tau_{n}}\right]
$$

where $\tau_{n}=l_{n}^{d / p} v_{n}\left(\left[n h_{n}\right]\right)^{d / 2}$. If $n$ is sufficiently large then $\tau_{n} \geq 2^{-d / 2} l_{n}^{d / p} v_{n}\left(n h_{n}\right)^{d / 2}$, hence using $h_{n} \geq n^{-\theta}$ we obtain $\tau_{n} \geq 2^{-d / 2}$. Finally, we derive

$$
\left\|A_{3, n}\right\|_{p}=\left\|\max _{1 \leq k \leq r_{n}} \mid V_{n}\left(x_{k}\right)\right\|\left\|_{p} \leq r_{n}^{1 / p} \sup _{x \in[0,1]^{d}}\right\| V_{n}(x) \|_{p}=O\left[v_{n}\right] .
$$

The proof of Lemma 5 is complete. The point $\mathbf{1}$ ) of Theorem 2 is obtained by combining inequality (18) and lemmas 2 and 5 .

Now, we are going to prove the point 2) of Theorem 2. We have

$$
\begin{aligned}
E\left(S_{n}(x)^{2}\right) & =\sum_{k, l \in \Lambda_{n}} a_{k}(x) a_{l}(x) E\left(\varepsilon_{k} \varepsilon_{l}\right) \\
& =\sum_{k \in \Lambda_{n}} a_{k}(x)^{2} E\left(\varepsilon_{k}^{2}\right)+\sum_{k \neq l} a_{k}(x) a_{l}(x) E\left(\varepsilon_{k} \varepsilon_{l}\right) \\
& =E\left(\varepsilon_{0}^{2}\right) \sum_{k \in \Lambda_{n}} a_{k}(x)^{2}+\sum_{k \in \Lambda_{n}} a_{k}(x) \sum_{l \in \Lambda_{n} \backslash\{k\}} a_{l}(x) E\left(\varepsilon_{k} \varepsilon_{l}\right) \\
& \leq \sum_{l \in \mathbb{Z}^{d}}\left|E\left(\varepsilon_{0} \varepsilon_{l}\right)\right| \times \sum_{k \in \Lambda_{n}} a_{k}(x) .
\end{aligned}
$$


If Condition C4) holds then using Lemma 1 there exists $\gamma>0$ such that for any $x \in[0,1]^{d}$ we have $E\left(S_{n}(x)^{2}\right) \leq \gamma \prod_{k=1}^{d}\left[n\left(x_{k}+h_{n}\right)\right]$. Let $x \in[0,1]^{d}$ be fixed, using Lemma 1, there exists $c>0$ such that

$$
\begin{aligned}
\left\|V_{n}(x)\right\|_{2} & =\frac{\left\|S_{n}(x)\right\|_{2}}{\sum_{i \in \Lambda_{n}} a_{i}(x)} \\
& \leq \frac{\sqrt{\gamma}}{c}\left(\prod_{k=1}^{d}\left[n\left(x_{k}+h_{n}\right)\right]\right)^{-1 / 2} \\
& \leq \frac{\sqrt{\gamma}}{c\left(\left[n h_{n}\right]\right)^{d / 2}} \\
& \leq \frac{2^{d / 2} \sqrt{\gamma}}{c\left(n h_{n}\right)^{d / 2}} \quad \text { for } n \text { sufficiently large. }
\end{aligned}
$$

The proof of Theorem 2 is complete.

\subsection{Proof of Proposition 1}

Since $g \in \operatorname{Lip}(B)$, it follows that

$$
\begin{aligned}
\left|B_{n}(x)\right| & =\left|\frac{\sum_{i \in \Lambda_{n}}(g(i / n)-g(x)) a_{i}(x)}{\sum_{i \in \Lambda_{n}} a_{i}(x)}\right| \\
& \leq B h_{n} \frac{\sum_{i \in \Lambda_{n}}\left\|(i / n-x) / h_{n}\right\| a_{i}(x)}{\sum_{i \in \Lambda_{n}} a_{i}(x)} \\
& \leq B h_{n} .
\end{aligned}
$$

The proof of Proposition 1 is complete.

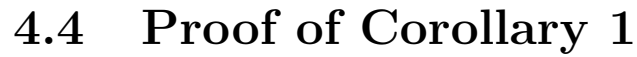

Let $h_{n}=\left(n^{-d} \log n\right)^{1 /(2+d)}$ then Proposition 1 gives

$$
\sup _{x \in[0,1]^{d}} \sup _{g \in \operatorname{Lip}(B)}\left|E g_{n}(x)-g(x)\right|=O\left[\left(\frac{\log n}{n^{d}}\right)^{\frac{1}{2+d}}\right] .
$$

Assume that C1) holds. Noting that

$$
\frac{(\log n)^{1 / 2}}{\left(n h_{n}\right)^{d / 2}}=\left(\frac{\log n}{n^{d}}\right)^{\frac{1}{2+d}}
$$


and using (5) we obtain

$$
\sup _{x \in[0,1]^{d}}\left|g_{n}(x)-E g_{n}(x)\right|=O_{a . s .}\left[\left(\frac{\log n}{n^{d}}\right)^{\frac{1}{2+d}}\right] .
$$

Combining (24) and (25) we derive (10).

Assume that C2) holds for some $0<q<2$. Noting that

$$
\frac{(\log n)^{1 / q}}{\left(n h_{n}\right)^{d / 2}}=\left(\frac{\log n}{n^{d}}\right)^{\frac{1}{2+d}} \times(\log n)^{(2-q) / 2 q}
$$

and using (6) we obtain

$$
\sup _{x \in[0,1]^{d}}\left|g_{n}(x)-E g_{n}(x)\right|=O_{a . s .}\left[\left(\frac{\log n}{n^{d}}\right)^{\frac{1}{2+d}} \times(\log n)^{(2-q) / 2 q}\right] \text {. }
$$

Combining (24) and (26) we derive (11).

Let $\varepsilon>0$ be fixed and assume that C3) holds for some $p>2$ which satisfies condition (12). Applying the point 3) of Theorem 11 with $\theta_{1}=1 /(2+d)$ and $\theta_{2}=d /(2+d)$ and noting that

$$
v_{n}=\frac{n^{a}(\log n)^{b}}{\left(n h_{n}\right)^{d / 2}}=n^{\varepsilon}\left(\frac{\log n}{n^{d}}\right)^{\frac{1}{2+d}} \Longleftrightarrow\left\{a=\varepsilon \text { and } b=\frac{1}{2}\right\}
$$

it follows

$$
\sup _{x \in[0,1]^{d}}\left|g_{n}(x)-E g_{n}(x)\right|=O_{a . s .}\left[n^{\varepsilon}\left(\frac{\log n}{n^{d}}\right)^{\frac{1}{2+d}}\right] .
$$

Combining (24) and (27) we derive (13). The proof of Corollary 1 is complete.

\subsection{Proof of Corollary 2}

Let $h_{n}=n^{-d /(2+d)}$ then Proposition 1 gives

$$
\sup _{x \in[0,1]^{d}} \sup _{g \in \operatorname{Lip}(B)}\left|E g_{n}(x)-g(x)\right|=O\left[n^{-\frac{d}{2+d}}\right] .
$$

Let $\varepsilon>0$ be fixed and assume that C3) holds for some $p>2$ which satisfies condition (14). Applying the point 1) of Theorem 2 and noting that

$$
v_{n}=\frac{n^{a}}{\left(n h_{n}\right)^{d / 2}}=n^{-\frac{d}{2+d}+\varepsilon} \Longleftrightarrow a=\varepsilon
$$


it follows that

$$
\left\|\sup _{x \in[0,1]^{d}}\left|g_{n}(x)-E g_{n}(x)\right|\right\|_{p}=O\left[n^{-\frac{d}{2+d}+\varepsilon}\right] .
$$

Combining (28) and (29) we derive (15).

Since $h_{n}=n^{-d /(2+d)}$ then $\left(n h_{n}\right)^{-d / 2}=h_{n}$. So, if C4) holds then combining (28) and (9) we derive (16). The proof of Corollary 2 is complete.

\subsection{Proof of Corollary 3}

Let $p>2$ be fixed. Using Rio's inequality [30] (see also Dedecker [13]) we obtain the bound

$$
\left\|\varepsilon_{k} E_{|k|}\left(\varepsilon_{0}\right)\right\|_{\frac{p}{2}} \leq 4\left(\int_{0}^{\alpha_{1, \infty}(|k|)} Q_{\varepsilon_{0}}^{p}(u) d u\right)^{2 / p}
$$

hence condition $\mathbf{C}^{\prime} \mathbf{3}$ ) is more restrictive than condition $\mathbf{C 3}$ ).

By Serfling's inequality (see McLeish 27] or Serfling [32]) we know that

$$
\left\|\varepsilon_{k} E_{|k|}\left(\varepsilon_{0}\right)\right\|_{\infty} \leq 2\left\|\varepsilon_{0}\right\|_{\infty}^{2} \phi_{\infty, 1}(|k|)
$$

so condition $\mathbf{C}^{\prime} \mathbf{1}$ ) is more restrictive than condition $\mathbf{C 1}$ ).

Now for $0<q<2$ there exists $C(q)>0$ (cf. Inequality (17) in [16]) such that

$$
\left\|\sqrt{\left|\varepsilon_{k} E_{|k|}\left(\varepsilon_{0}\right)\right|}\right\|_{\psi_{\beta(q)}}^{2} \leq C(q) \sqrt{\phi_{\infty, 1}(|k|)}
$$

In [16] we used the following lemma which can be obtain by the expansion of the exponential function.

Lemma 6 Let $\beta$ be a positive real number and $Z$ be a real random variable. There exist positive universal constants $A_{\beta}$ and $B_{\beta}$ depending only on $\beta$ such that

$$
A_{\beta} \sup _{p>2} \frac{\|Z\|_{p}}{p^{1 / \beta}} \leq\|Z\|_{\psi_{\beta}} \leq B_{\beta} \sup _{p>2} \frac{\|Z\|_{p}}{p^{1 / \beta}} .
$$

Consider the coefficient $c_{k}(\beta)$ given by (3) and denote

$$
d_{k}(p)=\left(\int_{0}^{\alpha_{1, \infty}(|k|)} Q_{\varepsilon_{0}}^{p}(u) d u\right)^{1 / p}
$$

then the following version of lemma 6 holds. 
Lemma 7 Let $\beta$ be a positive real number. There exist positive universal constants $A_{\beta}$ and $B_{\beta}$ depending only on $\beta$ such that for any $k \in \mathbb{Z}^{d}$

$$
A_{\beta} \sup _{p>2} \frac{d_{k}(p)}{p^{1 / \beta}} \leq c_{k}(\beta) \leq B_{\beta} \sup _{p>2} \frac{d_{k}(p)}{p^{1 / \beta}} .
$$

Now combining lemmas 6 and 7 and inequality (30) there exists $C^{\prime}(q)>0$ such that

$$
\left\|\sqrt{\left|\varepsilon_{k} E_{|k|}\left(\varepsilon_{0}\right)\right|}\right\|_{\psi_{\beta(q)}}^{2} \leq C^{\prime}(q) c_{k}^{2}(\beta(q)) .
$$

Finally condition $\mathbf{C}^{\prime} \mathbf{2}$ ) is more restrictive than condition $\mathbf{C 2}$ ) and the proof of Corollary 3 is complete.

Aknowledgements. I would like to express my thanks to the anonymous referee for his/her careful reading of the manuscript and valuable suggestions. I am indebted for É. Youndje for many stimulating conversations on nonparametric estimation.

\section{References}

[1] K. S. Alexander and R. Pyke. A uniform central limit theorem for set-indexed partial-sum processes with finite variance. Ann. Probab., 14:582-597, 1986.

[2] N. Altman. Krige, smooth, both or neither ? Australian and New Zealand Journal of Statistics, 42:441-461, 2000.

[3] R. F. Bass. Law of the iterated logarithm for set-indexed partial sum processes with finite variance. Z. Wahrsch. verw. Gebiete, 70:591-608, 1985 .

[4] A. K. Basu and C. C. Y. Dorea. On functional central limit theorem for stationary martingale random fields. Acta. Math. Hung., 33:307-316, 1979 .

[5] G. Biau. Spatial kernel density estimation. To appear in Mathematical methods of Statistics, 2003.

[6] G. Biau and B. Cadre. Nonparametric spatial prediction. To appear in Statistical Inference for Stochastic Processes, 2003.

[7] E. Bolthausen. Exact convergence rates in some martingale central limit theorems. Ann. Probab., 10(3):672-688, 1982. 
[8] D. Bosq. Bernstein-type large deviations inequalities for partial sums of stron mixing processes. Statistics, 24:59-70, 1993.

[9] D. Bosq. Nonparametric Statistics for Stochastic Processes-Estimation and Prediction-2nde Edition. Lecture Notes in Statistics, Springer Verlag, New York, 1998.

[10] M. Carbon, M. Hallin, and B. Wu. Kernel density estimation for random fields: the $l_{1}$ theory. Nonparametric Statist., 6:157-170, 1996.

[11] M. Carbon, L.T. Tran, and B. Wu. Kernel density estimation for random fields. Statist. Probab. Lett., 36:115-125, 1997.

[12] J. Dedecker. A central limit theorem for stationary random fields. Probab. Theory Relat. Fields, 110:397-426, 1998.

[13] J. Dedecker. Exponential inequalities and functional central limit theorems for random fields. ESAIM: Probability and Statistics, 5:77-104, 2001.

[14] R. L. Dobrushin. The description of a random fields by mean of conditional probabilities and condition of its regularity. Th. Probab. Appl., 13:197-224, 1968.

[15] P. Doukhan. Mixing : Properties and Examples, volume 85. Lecture Notes in Statistics, Berlin, 1994.

[16] M. El Machkouri. Kahane-Khintchine inequalities and functional central limit theorem for stationary random fields. Stoch. Proc. and Their Appl., 120:285-299, 2002.

[17] M. El Machkouri and D. Volný. Contre-exemple dans le théorème central limite fonctionnel pour les champs aléatoires réels. Annales de l'IHP, 2:325-337, 2003.

[18] X. Guyon. Random fields on a Network: Modeling, Statistics and Applications. Springer, New York, 1995.

[19] P. Hall and J.D. Hart. Nonparametric regression with long-range dependence. Stoch. Proc. and Their Appl., 36:339-351, 1990.

[20] M. Hallin, Z. Lu, and L.T. Tran. Density estimation for spatial linear processes. Bernoulli, 7:657-668, 2001. 
[21] M. Hallin, Z. Lu, and L.T. Tran. Density estimation for spatial processes: the $l^{1}$ theory. Journal of Multivariate Analysis, 88:61-75, 2004.

[22] M. Hallin, Z. Lu, and L.T. Tran. Local linear spatial regression. Annals of Statistics, 32, 2004. In press.

[23] I. A. Ibragimov. Some limit theorems for stationary processes. Theory Probab. Appl., 7:349-382, 1962.

[24] M. A. Krasnosel'skii and Y. B. Rutickii. Convex Functions and Orlicz Spaces. P. Noordhoff LTD-Groningen-The Netherlands, 1961.

[25] Z. Lu and X. Chen. Spatial nonparametric regression estimation: Nonisotropic case. Acta Mathematicae Applicatae Sinica, English series, 18:641-656, 2002.

[26] Z. Lu and X. Chen. Spatial kernel regression estimation: weak consistency. Statistics and Probability Letters, 68:125-136, 2004.

[27] D. L. McLeish. A maximal inequality and dependent strong laws. Ann. Probab., 3(5):829-839, 1975.

[28] B. Nahapetian. Limit Theorems and Some Applications in Statistical Physics. B. G. Teubner Verlagsgesellschaft, Stuttgart, Leipzig, 1991.

[29] B. Nahapetian and A. N. Petrosian. Martingale-difference Gibbs random fields and central limit theorem. Ann. Acad. Sci. Fenn., Series A-I Math., 17:105-110, 1992.

[30] E. Rio. Covariance inequalities for strongly mixing processes. Annales de l'IHP, 29(4):587-597, 1993.

[31] M. Rosenblatt. A central limit theorem and a strong mixing condition. Proc. Nat. Acad. Sci. USA, 42:43-47, 1956.

[32] R. J. Serfling. Contributions to central limit theory for dependent variables. Ann. Math. Statist., 39(4):1158-1175, 1968.

[33] C. J. Stone. Optimal global rates of convergence for nonparametric regression. Annal. of Statist., 10(4):1043-1053, 1982.

[34] L.T. Tran. Kernel density estimation on random fields. Journal of Multivariate Analysis, 34:37-53, 1990.

[35] L.T. Tran and Yakowitz S. Nearest neighbor estimators for random fields. Journal of Multivariate Analysis, 44:23-46, 1993. 
[36] Q. Yao. Exponential inequalities for spatial processes and uniform convergence rates for density estimation. In Development of Modern Statistics and Related Topics - In Celebration of Prof. Yaoting Zhang's 70th Birthday, H. Zhang and J. Huang (edit.), World Scientific, Singapore, pages 118-128, 2003.

Mohamed EL MACHKOURI

Laboratoire de Mathématiques Raphaël Salem

UMR 6085, Université de Rouen

Site Colbert,

F76821 Mont-Saint-Aignan Cedex

email : mohamed.elmachkouri@univ-rouen.fr 